\title{
Interfaces in driven Ising models: shear enhances confinement
}

\author{
Thomas H. R. Smith, ${ }^{1}$ Oleg Vasilyev, ${ }^{2}$ Douglas B. Abraham,,${ }^{2,3}$ Anna Maciołek, ${ }^{2,3,4}$ and Matthias Schmidt ${ }^{1}$ \\ ${ }^{1}$ H.H. Wills Physics Laboratory, University of Bristol, \\ Tyndall Avenue, Bristol BS8 1TL, United Kingdom \\ ${ }^{2}$ Max-Planck-Institut für Metallforschung, Heisenbergstraße 3, D-70569 Stuttgart, Germany \\ ${ }^{3}$ Theoretical Physics, Department of Physics, University of Oxford, \\ 1 Keble Road, Oxford OX1 3NP, United Kingdom \\ ${ }^{4}$ Institute of Physical Chemistry, Polish Academy of Sciences, \\ Department III, Kasprzaka 44/52, PL-01-224 Warsaw, Poland
}

(Dated: 12 June 2008)

\begin{abstract}
We use a phase-separated driven two-dimensional Ising lattice gas to study fluid interfaces exposed to shear flow parallel to the interface. The interface is stabilized by two parallel walls with opposing surface fields and a driving field parallel to the walls is applied which (i) either acts locally at the walls or (ii) varies linearly with distance across the strip. Using computer simulations with Kawasaki dynamics, we find that the system reaches a steady state in which the magnetization profile is the same as that in equilibrium, but with a rescaled length implying a reduction of the interfacial width. An analogous effect was recently observed in sheared phase-separated colloidal dispersions. Pair correlation functions along the interface decay more rapidly with distance under drive than in equilibrium and for cases of weak drive can be rescaled to the equilibrium result.
\end{abstract}

PACS numbers: 05.40.-a, 05.50.+q, 68.05.Cf, 68.35.Rh

Shearing a fluid is a fundamental example in the statistical mechanics of systems driven away from equilibrium [1. Profound effects on the liquid structure [2] and new phase transitions [3] can result. In a recent intriguing advance in colloidal science interfacial fluctuations of capillary wave-type were observed by direct optical means [4]; these fluctuations can be strongly supressed by shearing in the direction parallel to the interface [5].

Kinetic Ising models which have been driven out of equilibrium are of continuing importance in condensed matter physics 6, 7, 8. Underlying is the idea of coarse graining in time [9], where a record of the dynamical state is only kept at discrete points in time, which is an approximation that can often be justified by the quality and range of deductions made. Mass is conserved, but there is no inertia and associated hydrodynamics. Nevertheless currents are predicted as well as densities. Examples showing the value of such stochastic models include studies of interfaces under spatially homogeneous and random driving fields [7], domain growth [8] and nucleation [10] under shear, as well as of the precursor film in complete wetting [11].

In this Letter, we investigate the effects of shear on the interface between coexisting phases using the twodimensional $(2 d)$ Ising lattice gas with Kawasaki dynamics [12. The interface is induced by fixing spins on the boundaries of a strip; the analogue of shearing in a lattice gas will be explained below. Studying the $2 d$ problem is interesting in its own right, because in equilibrium the interface is known to be rough for all temperatures $T$ below the bulk critical temperature $T_{c}$. If the interface is confined to a strip of width $L_{y}$, then it sweeps out essentially the entire strip, meandering back and forth between the walls, such that the interfacial width $w \sim L_{y}$, despite the entropic repulsion from the walls [13. Consequently, at fixed $T$, the magnetization profile as a function of distance $y$ across the strip scales as $m_{\mathrm{b}}(T) \mathcal{M}_{\text {eq }}\left(y / L_{y}\right)$, where $m_{\mathrm{b}}(T)$ is the spontaneous magnetization in bulk and $\mathcal{M}_{\mathrm{eq}}$ is the finite size scaling function for a strip with size $L_{x}\left(\gg L_{y}\right)$ along the walls; "eq" labels equilibrium quantities.

Using Monte Carlo (MC) simulations we find that the $2 d$ Ising model under shear-like drive parallel to the interface achieves a steady state that is characterized by an effective length scale $L_{y}^{\star}$, such that the magnetization profile $m(y)$ at given $T$ obeys

$$
\frac{m(y)}{m_{\mathrm{b}}(T)} \approx \mathcal{M}_{\mathrm{eq}}\left(\frac{y}{L_{y}^{\star}}\right)+\mathcal{M}_{\text {corr }}(y) \quad \text { with } \quad L_{y}^{\star}<L_{y}
$$

where $\mathcal{M}_{\text {corr }}(y)$ is a boundary correction term, which decays away from the boundaries on the scale of the bulk correlation length $\xi$ (on which the exponential decay of spin-spin correlations occurs in the homogeneous system). A conjecture based on Eq. (1) is that shear-like drive acts as effective confinement of the system.

To be specific, in spin language the Hamiltonian is $H=-J \sum_{<i, j>} \sigma_{i} \sigma_{j}$, where the sum runs over nearest neighbour sites $i, j ; J>0$ is the spin-spin coupling constant, and the spins take on values $\sigma_{i}= \pm 1$, corresponding to particle occupation numbers $\tau_{i}=\left(\sigma_{i}+1\right) / 2=0,1$. The interface is induced and localized by two walls of fixed spins $\sigma_{i}=+1$ at the top $\left(y=L_{y}+1\right)$ and $\sigma_{i}=-1$ at the bottom $(y=0)$ edges of the strip. Periodic boundary conditions are applied in the $x$-direction. In order to induce shear, we apply a force field $J F(y)$ parallel to the $x$-direction. In model I only the particles in the layer adjacent to each wall are driven along the walls and in opposite directions: $F\left(L_{y}\right)=F_{0}$ and $F(1)=-F_{0}$, and 
$F(y)=0$ otherwise; for $F_{0} \rightarrow \infty$ these layers form an asymmetric exclusion processes [6, coupled to an Ising strip. In model II the force varies linearly across the strip, such that it vanishes in the middle of the slit: $F(y)=$ $\omega\left[y-\left(L_{y}+1\right) / 2\right]$, where $\omega=\partial F(y) / \partial y$ is the (dimensionless) field difference between adjacent rows; we also use a scaled variable $\tilde{y}=\left(2 y-L_{y}-1\right) / L_{y}$, see Fig. 11a. Model II can be thought of as mimicking the effects caused by the flow of a background solvent [5]. The system evolves under spin-exchange Kawasaki dynamics [12, corresponding to particle hopping to an (empty) nearest neighbour site. The work done by (or against) the force field, $\Delta F$, in a trial move enters a modified Metropolis acceptance rate, $\min \left\{1, \exp \left(-(\Delta H+\Delta F) /\left(k_{B} T\right)\right)\right\}$, where $\Delta H$ is the change in internal energy and $k_{B}$ is the Boltzmann constant. The dynamics capture the local conservation of particle number and the competition of forced transport with diffusive motion.

We have performed extensive simulations using singlespin and multi-spin coding techniques [14, the latter generalized to include drive; this facilitates simultaneously running 64 systems per processor core. We present here results for $L_{x}=200$ and $L_{y}$ varying from 10 to 40 at fixed total magnetization $m=\sum_{i} \sigma_{i}=0$ for $T / T_{c}=$ $0.75,0.85$, and 0.95 (where $T_{c}=2.2619 \mathrm{~J} / k_{B}$ ), so that $L_{y} / \xi>1$. Sampling large-scale interfacial fluctuations with inherently slow Kawasaki dynamics is challenging and requires run lengths of the order of $N_{\mathrm{MC}}=10^{8}$ MC steps $\left(L_{x} \times L_{y}\right.$ trial moves form one MC step). For each run we perform initially $N_{\mathrm{MC}} \mathrm{MC}$ steps and find that thereafter all observables of interest fluctuate around their mean value. We conclude that a steady state is reached, in which we gather statistics for a further $N_{\mathrm{MC}}$ steps. Statistical errors are obtained with blocking and bootstrap techniques. Fig. 1 displays snapshots of configurations [15.

Driving creates a non-vanishing current profile $j(y)$ parallel to the walls; $j(y)$ gives the net (mean) number of particles that move from $x$ to $x+1$ at given $y$ in one MC step. For the boundary-driven case (model I) the current is localized at both walls, $y=1, L_{y}$, and vanishes in the middle of the system (apart from a very small anti-current at $\left.y=2, L_{y}-1\right)$. In the bulk-sheared case (model II) $j(\tilde{y})$ varies smoothly with $\tilde{y}$, see Fig. 11. For small values of $\omega$ a near-linear behaviour is observed. For strong drive the current saturates upon approaching the walls. Surprisingly $|j(\tilde{y})|$ displays pronounced maxima offset from the center of the strip, indicating an optimum between sufficiently strong drive and sufficiently large number of particle-hole pairs.

Having established the occurence of shear flow, we investigate the interfacial magnetization profile $m(y)=$ $L_{x}^{-1}\left\langle\sum_{x} \sigma(x, y)\right\rangle$, where the angles denote an average in the steady state. We have checked that the aspect ratio $L_{x} / L_{y}$ is large enough such that at equilibrium the exact result [13] for $m(y)$, for $L_{x} \rightarrow \infty$, is indistinguish-
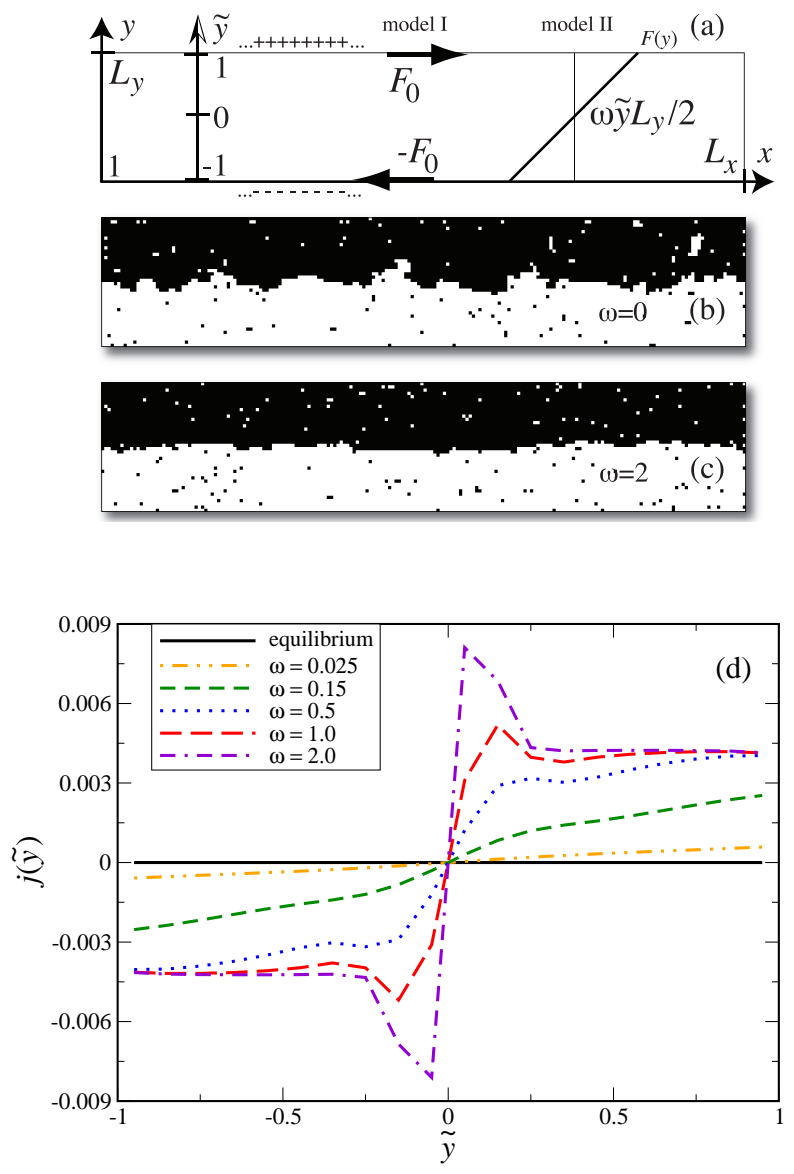

FIG. 1: (color online) a) Illustration of the $2 d$ Ising strip between $+/$ - walls under an external driving field $J F(y)$ parallel to the walls. The field acts with strength $F_{0}$ at the boundary layers only (model I) or varies linearly with (scaled) distance $\tilde{y}$ across the strip, where $\omega$ determines the slope (model II). b) Snapshot from simulation for $T / T_{c}=0.75, L_{x}=200$, and $L_{y}=20$ at equilibrium; black (white) regions indicate $\sigma_{i}=+1(-1)$. c) Same as b) but under strong drive according to model II with $\omega=2$. d) The current profile $j(\tilde{y})$ parallel to the walls as a function of $\tilde{y}$ for different values of $\omega$ (as indicated) in model II for the same values of $T, L_{x}, L_{y}$ as in b.

able within statistical errors from our simulation results (not displayed) using Glauber dynamics [16, where $m$ can fluctuate. Using Kawasaki dynamics (that constrain $m=0$ ) leads to larger flat regions near the walls and a sharper interfacial region, see Fig. 20 ; this effect increases strongly if $L_{y} / \xi \gg 1$. The importance of the contribution of capillary-wave type fluctuations to $m(y)$ can be gauged by the stark contrast to the kink-like behaviour obtained in equilibrium mean-field theory. For cases of weak boundary drive (small values of $F_{0}$ ) the magnetization near the walls increases and the interfacial region gets squeezed (becomes narrower) upon increasing $F_{0}$; this behaviour is more apparent for higher values of $T$. For $F_{0} \gtrsim 5$ there are no further changes in the pro- 

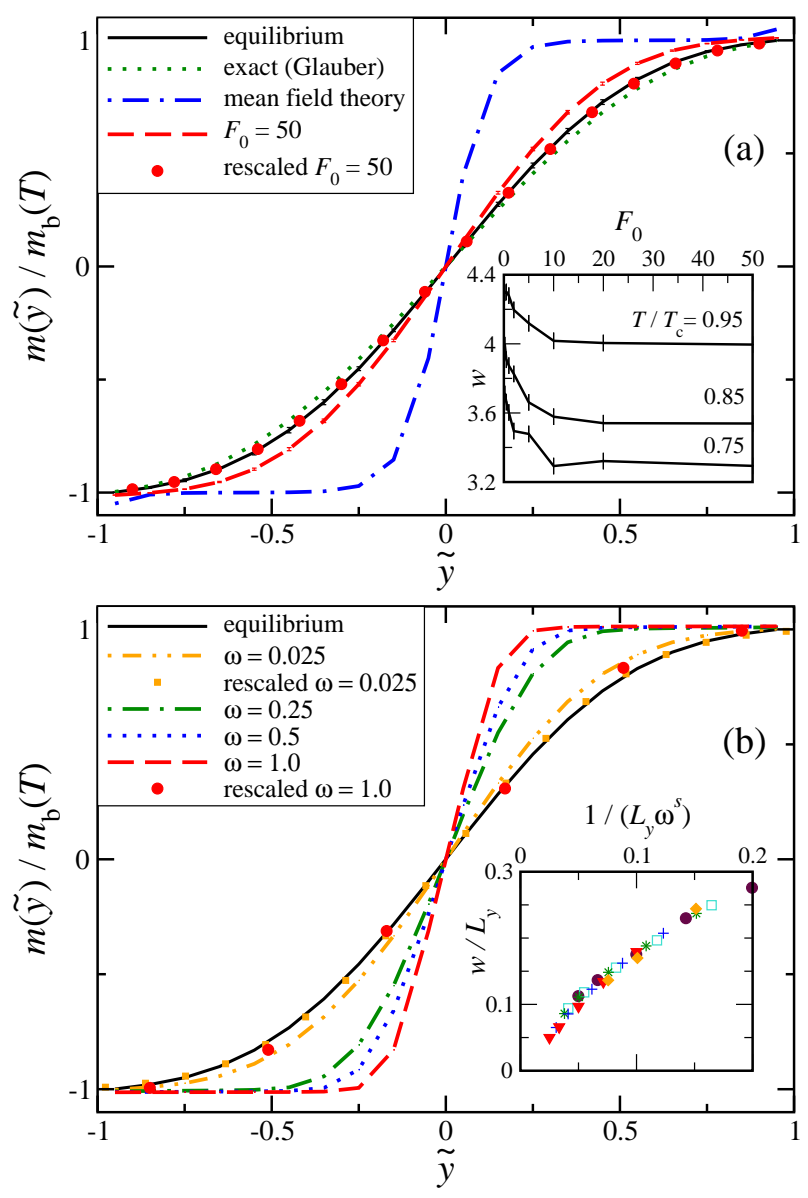

FIG. 2: (color online) Scaled magnetization profiles $m(\tilde{y}) / m_{\mathrm{b}}(T)$ as a function of the scaled distance $\tilde{y}$ for $T / T_{c}=$ $0.75, L_{x}=200, L_{y}=20$. a) Model I: Kawasaki equilibrium result, Glauber equilibrium result, mean-field result, Kawasaki result for $F_{0}=50$, and the latter rescaled as $m(0.83 \tilde{y})$. Inset: Variation of the interfacial width $w$ with $F_{0}$ for three different temperatures (as indicated). b) Kawasaki simulation results for model II are shown in equilibrium and for a range of values of the field gradient $\omega$ (as indicated), as well as using rescaling $m\left(a_{\perp} \tilde{y}\right)$, where $a_{\perp}=1 / 1.15,1 / 3.4$ for $\omega=0.025,1$, respectively. Error bars are of the order of the line thickness. Inset: Variation of the scaled interfacial width $w / L_{y}$ with the scaling variable $1 /\left(L_{y} \omega^{s}\right)$, where $s=0.3$, for $\omega=0.025$ (diamonds), 0.1 (filled circles), 0.2 (squares), 0.25 (stars), 0.5 (crosses), and 1 (triangles).

file within error bars; in Fig. $2 \mathrm{a}$ the limiting behaviour, $F_{0}=50$, is shown. The effect on $m(y)$ arises from a competition of advection and diffusion: the boundary drive helps to break up clusters into smaller constituents; these possess high mobility and are hence able to migrate towards and coalesce with their bulk phase for energetic reasons. This dynamic mechanism makes the observed additional confinement of the interface physically reasonable. The effect of the boundary drive cannot be mimicked by assuming equilibration in static boundary fields acting at $y=2$ and $L_{y}-1$. Even infinitely strong surface fields $h(y=1)=-h\left(y=L_{y}\right)=\infty$ induce a significantly weaker effect. Particularly striking is the scaling behavior of $m(y)$, see Eq. (1), which indicates that the effect of the boundary drive on the interfacial region of the profile is the same as that induced by increasing the confinement, and hence reducing the width of the strip from $L_{y}$ to $L_{y}^{\star}=0.83 L_{y}$ for $F_{0} \gtrsim 5$. This corresponds to the scaling $m\left(a_{\perp} \tilde{y}\right) \approx m_{\mathrm{eq}}(\tilde{y})$, where for strong boundary drive (such that essentially $F_{0} \rightarrow \infty$ ) we find $a_{\perp}=L_{y}^{\star} / L_{y}=0.83$ for $L_{x}=200$ and all values of $T$ and $L_{y}$ considered. We expect that $w \sim L_{y}^{\star}$, which we have checked explicitly by calculating $w$ as the second moment of $\partial m(y) / \partial y$, showing that $w$ decreases rapidly with $F_{0}$ and eventually saturates at a finite value, see the inset in Fig. 2a. Model II displays similar squeezing of the interfacial region, see Fig. 2 p. For small values of $\omega$ the effect is very similar to the boundary-driven case. Increasing $\omega$ leads to very pronounced squeezing, such that the central section of the profile for $\omega=1$ resembles the mean-field solution in equilibrium! Even this case can be rescaled to the equilibrium profile, see Fig. 2 2 . We find that the variation of $w$ with transversal system size and field strength can be condensed into dependence on the scaling variable $1 /\left(L_{y} \omega^{s}\right)$ for any value of $T$ considered; for $T / T_{c}=0.75$ data collapse is achieved for $s=0.3$, see the inset of Fig. 2b. Similar quality of scaling can be obtained by including $\left(T-T_{c}\right) / T_{c}$ in the scaling variable [7; these results (not shown) indicate that in cases of weak drive $a_{\perp}=L_{y}^{\star} / L_{y}$ increases (and hence the effective confinement becomes weaker) with increasing $T$.

We next investigate the interfacial structure on the two-body level and consider the spin-spin pair correlation function in steady-state $G\left(x, y, y^{\prime}\right)=\left\langle\sigma_{i} \sigma_{j}\right\rangle$, where $i=(0, y)$ and $j=\left(x, y^{\prime}\right)$, focusing on the behaviour along the center, $G_{\|}(x) \equiv G\left(x, L_{y} / 2, L_{y} / 2\right)$; this should reveal most clearly interface-mediated correlations. The simulation results shown in Fig. $3 \mathrm{a}$ indicate that shear induces a faster decay of $G_{\|}(x)$ with distance $x$ than at equilibrium. For model I and for weak drive in model II, the behaviour at short and intermediate distances can be accurately described by rescaling the lateral coordinate, $G_{\|}\left(a_{\|} x\right) \approx G_{\|}^{\mathrm{eq}}(x)$. From fitting the data we find the ratio of the lateral (interfacial) corrrelation lengths in and out of equilibrium, $a_{\|}=\xi_{\|} / \xi_{\|}^{\mathrm{eq}}<1$, see Fig. 3. Unlike the behaviour on the one-body level, $G_{\|}(x)$ under strong drive in model II does not resemble the equilibrium meanfield result (obtained by calculating the inhomogeneous magnetization around a fixed spin in the interface), which displays significantly longer-ranged decay.

We studied coarse-grained interfacial properties via the local position (height) of the interface $h(x)$, obtained either following [17] or based on the (scaled) column magnetization: $h(x)=\left(2 m_{\mathrm{b}}\right)^{-1} \sum_{y} \sigma(x, y)$; both methods give consistent results. Fig. $3 \mathrm{~b}$ displays results for the 

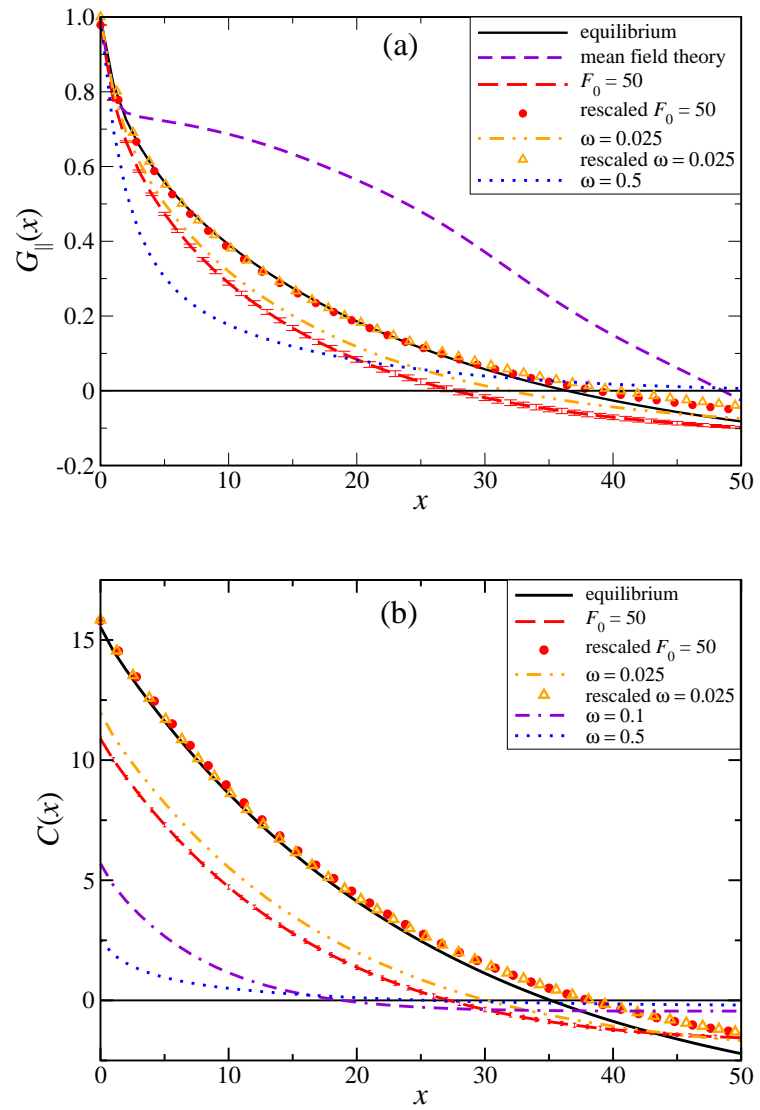

FIG. 3: (color online) Spin-spin pair correlation function $G_{\|}(x)$ at the center of the strip (a) and height-height correlation function $C(x)$ (b) as a function of distance $x$ for $T / T_{c}=0.75, L_{x}=200, L_{y}=20$. Shown are results in equilibrium, from mean-field theory (only for $G_{\|}(x)$ in a), from Kawasaki simulations with strong boundary-drive in model I $\left(F_{0}=50\right)$, the same rescaled as $G_{\|}(x / 1.4)$; Kawasaki simulation results for model II are shown for $\omega=0.025,0.1$ (only for $C(x)$ in b) and 0.5 , as well as the former case rescaled as $G_{\|}(x / 1.27)$.

height-height correlation function $C(x)=\langle h(x) h(0)\rangle$; this is directly related to the spin-spin correlation function: $C(x)=\left(4 m_{\mathrm{b}}^{2}\right)^{-1} \sum_{y, y^{\prime}} G\left(x, y, y^{\prime}\right)$. The intercept, $C(0)=\left\langle h(0)^{2}\right\rangle$, provides an alternative measure of the (squared) interfacial width; we find that $C(0)$ decreases under shear, very similarly to the behaviour of $w^{2}$, exhibiting a very strong effect for large values of $\omega$. $C(x)$ decays faster with $x$ than in equilibrium, in agreement with the behaviour of the spin-spin correlation function. Hence from the scaling of $m(y)$ and $G_{\|}(x)$, we expect $a_{\perp}^{-2} C\left(a_{\|} x\right) \approx C_{\mathrm{eq}}(x)$, in correspondence with Weeks scaling in equilibrium [18: $C_{\text {eq }}(x) \approx w^{2} \mathcal{C}\left(x / \xi_{\|}^{\mathrm{eq}}\right)$, where $\mathcal{C}$ is the scaling function, and $w \sim L_{y}$. Indeed both for strong boundary drive and weak bulk drive data collapse is achieved using the same values for $a_{\|}$and $a_{\perp}$ as obtained above.
Comparing our findings for $C(x)$ to experimental results for the height-height correlation function of a phaseseparated colloid-polymer mixture, Fig. 3 of Ref. [5], reveals a striking similarity in the reduction of the amplitude of the correlation function under shear. This is consistent with the observation of a reduction of the interfacial roughness in both studies. However, Derks et al. find an increase of the lateral correlation length under shear (Fig. 4b of [5]), as obtained from fitting the correlation function of the equilibrium capillary wave model to the steady state data. This finding is in contrast to the behaviour of our models. Despite the differences in the way the interface is localized (whether experimentally by gravity or here by walls), the fundamental question arises as to which features of interfaces under shear are universal. Clearly, dimensionality is expected to play a major role. The $3 d$ Ising model has a rough phase above the (finite) roughening transition temperature. However, the roughness of the interface is established by a very different mechanism than in $2 d$, in that the interface throws out spikes [19], which are expected to hinder fluid flow to a much lesser degree than interface meandering in $2 d$. This should have important repercussions on the interfacial structure under drive.

We thank D. Aarts, D. Derks, J. Eggers, R. Evans, A. Gambassi and R. Zia for useful discussions and the EPSRC for support.

[1] R.M.L. Evans, Phys. Rev. Lett. 92, 150601 (2004).

[2] O. Henrich et al., J. Phys.: Condens. Matter 19, 205132 (2007); J.M. Brader et al., Phys. Rev. Lett. 98, 058301 (2007). M.P. Lettinga et al., Phys. Rev. E 70, 061405 (2004).

[3] A. Onuki, J. Phys.: Condens. Matter 9, 6119 (1997).

[4] D.G.A.L. Aarts et al., Science 304, 847 (2004).

[5] D. Derks, D.G.A.L. Aarts, D. Bonn, H.N.W. Lekkerkerker, and A. Imhof, Phys. Rev. Lett. 97, 038301 (2006).

[6] B. Schmittmann and R.K.P. Zia, in Phase Transitions and Critical Phenomena, ed. by C. Domb and J.L. Lebowitz, Vol. 17 (Academic, London, 1995), p.1.

[7] K.-t. Leung, K.K. Mon, J.L. Vallés, and R.K.P. Zia, Phys. Rev. B 39, 9312 (1989); K.-t. Leung and R.K.P. Zia, J. Phys. A: Math. Gen. 26, L737 (1993).

[8] E.N.M. Cirillo et al., Phys. Rev. E 72, 026139 (2005).

[9] O. Penrose, Foundations of Statistical Mechanics, (Pergamon Press Ltd., Oxford 1970).

[10] R.J. Allen et al., arXiv:0805.3029.

[11] D.B. Abraham et al., Phys. Rev. Lett. 88, 206101 (2002).

[12] K. Kawasaki, Phys. Rev. 145, 145 (1966).

[13] J. Stecki et al., Phys. Rev. B 49, 1092 (1994).

[14] S. van Gemmert et al. Phys. Rev. E 72, 046131 (2005).

[15] See the accompanying EPAPS material for movies.

[16] R. Glauber, J. Math. Phys. 4, 294 (1963).

[17] A. De Virgiliis, E.V. Albano, M. Müller, and K. Binder Physica A 352, 477 (2005).

[18] D. Bedeaux and J.D. Weeks, J. Chem. Phys. 82, 972 (1985).

[19] J. Bricmont et al., J. Stat. Phys. 42, 743 (1986). 